\title{
Dynamics of trunk type robot with spherical piezoelectric actuators
}

\author{
Aistis Augustaitis ${ }^{1}$, Vytautas Jurènas ${ }^{2}$ \\ ${ }^{1}$ Department of Informational Technologies, Vilnius Gediminas Technical University, Lithuania \\ ${ }^{2}$ Institute of Mechatronics, Kaunas University of Technology, Lithuania
}

\begin{tabular}{l}
\hline \hline Article Info \\
\hline Article history: \\
Received Feb 7, 2020 \\
Revised Feb 24, 2020 \\
Accepted Mar 10, 2020 \\
\hline
\end{tabular}

\section{Keywords:}

Euler-lagrange dynamics

Kinematics

Potential torque

Spherical piezoactuator

\begin{abstract}
Trunk type robots (TTRs) are exclusive. These robots can provide a high level of maneuverability and have a potential in medicine or high risk zones. TTRs are determined as a long serial linkage of similar segments. They are usually connected using tendons or small actuators. A spherical actuator is the most appreciable option. The motion of real spherical actuator (RSA) can be easily obtained applying an inverse piezoelectric effect. It has three independent spinning axes. These axes are perpendicular to each other despite the history of excitation. Kinematics and dynamics of RSA almost have no basics regardless of mentioned features. This situation can be explained according to common disadvantages of other SAs: sophisticated structure and complex control. The structures and abilities of TTRs are reviewed in the first section of this article. At the beginning of the fourth section the kinematics of piezoelectric TTR with two different RSAs is introduced. Its results of inverse dynamics using Euler-Lagrange equations are presented at the end of the fourth section. Similar results are derived using an analytical-potential method in the fifth section. It is quite accurate and effective option to determine inverse dynamics of the TTR employing an analytical-potential method.
\end{abstract}

This is an open access article under the CC BY-SA license.

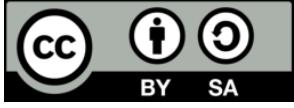

\section{Corresponding Author:}

Aistis Augustaitis,

Department of Informational Technologies,

Vilnius Gediminas Technical University,

Saulètekio al. 11, Vilnius 10223, Lithuania.

Email: ais.augustaitis@gmail.com

\section{INTRODUCTION}

A trunk type robot (TTR) is a serial chain of links. They can be connected with tendons or flexible and rotary actuators. A real spherical actuator (RSA) has three perpendicular spinning axes and this state is constant despite the history of excitation. A spherical actuator usually has very sophisticated structure and is hard to control accurately. Consistently a RSA is very unpopular in practical and theoretical cases.

Grasping abilities of TTR are investigated by Li, Teng, Xiao, Kapadia, Bartow and Walker in 2D workspace. The robot is autonomous and consists of flexible pneumatic actuators, but has no end effector and closed loop system too. The numerical operations for target detection and path determination take only $0.2 \mathrm{~s}$. The time of manipulation ranges from 30 to 130 s seeking to avoid unwelcome oscillations because of low stiffness and high inertia [1].

A pneumatic robot is introduced by Mahl, Hildebert and Sawodny. It consists of 3 serial stages and a three-fingered end effector. Three evenly placed flexible hoses in circumference direction are used to control one stage. Every stage can be bended independently. A total bending moment grows up from the end point to the first link of the robot, so its cross-sections are enlarged in the same way [2]. 
Nagarajan, Kumar and Kanna are made a direct kinematic research for a TTR with tendons using ADAMS and ANSYS modeling systems. They have selected to explore the robot with four independent sections. Every section has 6 serially connected disks and universal joints between them. One tendon is able to transfer the force only in one direction. Therefore two tendons are needed to ensure an actuation of single DOF. Four evenly posted tendons are used to actuate a single section. Four evenly posted springs has been employed for every pair of disks to ensure the stiffness of the robot. The authors have proved again that deformations between the disks of the same section are distributed uniformly [3].

A special design of soft robot to mimic the motions of elephant trunk or snake slithering is generated. The robot is a combination of few sections. Each section is a group of tubular and helical segments. They are made from the strips using ionic polymer-metal nanocomposites (IPMCs). This type of segments are able to perform not only various actuations (linear expansion, contraction, bending and twisting), but energy harvesting and motion sensing too [4].

Jones and Walker have presented a kinematic theory for a TTR with flexible links. It can be actuated employing different air pressures in gofferred hoses or different lengths of tendons too. The estimation of position and orientation and a brief report about its singularities are described comprehensively. The local parameters of pressures or lengths and their first or second derivatives can be estimated according to inverse Jacobian matrix [5].

Chung, Rhee, Shim, Lee and Park have analyzed door opening robots. These robots can be used in emergency situations to shift humans. These authors have offered a new conception for that kind of robots. They suggest a manipulator with three fingers and the force sensors at their tips as an alternative for a wrist sensor. It has a high price and a complex structure too. A tested robot can be separated into 3 motion stages: omnidirectional chassis, manipulator with 6 degrees of freedom (DOFs) and an end effector with 3 fingers. These stages are actuated by 14 servomotors. 16 sonar, 16 infrared sensors and a laser range finder are installed to enhance the features of robot vision [6].

A TTR of serially connected tubes with inclined end planes at one side is presented by Salomon and Wolf. It is able to perform 16 degrees of freedom. The same type planes of the tubes are conjugated together. A revolute actuator goes after cylindrical-incline actuator and so on. This robot provides high level features like stiffness and position accuracy. It possesses these dimensions: diameter of tube is $7.7 \mathrm{~cm}$, total length $-80 \mathrm{~cm}$ and a maximum deviation angle $180^{\circ}$. A maximum traverse force at the end of the robot should be less than $25 \%$ of its own weight [7].

The abilities of motion are investigated and tested by Liljebäck, Haugstuen and Pettersen for a snake type robot (STR). This robot is composed of 10 spherical segments. They are serially connected with short flexible links. A STR has a similar structure according to a TTR. The rotating frame with crosswise oriented wheels around it is attached to every segment. This way the action of moving straight and transversely is ensured for every segment. A proper control of the robot is achieved using an internal function ode 45 in MATLAB software. A combined task of STR is to detect a straight cursor line, change its direction and crawl along the line. The task has been successfully implemented using a novel stabilization algorithm [8].

Kelasidi, Jesmani, Pettersen and Gravdahl have introduced a multi-objective optimization framework. This method is applied to investigate the efficiency of locomotion for snake type robots employing a weighted sum method. A Particle Swarm Optimization (PSO) is used for different set of weight factors. The consumed power of the robot and its forward velocity are selected as the main parameters of research. Consistently an improved energy efficiency of locomotion associates with the decrement of forward velocity [9].

Important processes of redundant robots like motion, recognition of objects, fetching and safe interface with humans have been widely investigated by Luo and Kuo. A service-oriented multiagent system (SoMAS) is used to control and analyze the robot with cyber-physical system (CPS). The results of mentioned operations approve the effectiveness of integrated systems regarding to high position accuracy and fast speed. Other high quality factors of the robot in comparison to the results of other researchers are mentioned too [10].

Several piezoeletric robots are presented in Figures 1 and 2. They can be named as trunk type robots and have been made in Mechatronics Institute of KTU. Piezoelectric TTRs are special because of small dimensions (total length usually is less than $20 \mathrm{~cm}$ ) and high ratio of output power to mass. They possess a simple structure and a high accuracy of position too. These TTRs can be significant in medicine or high risk zones. An extra freedom to manoeuvre is achieved employing a piezoelectric spherical actuator with 3 independent spinning DOFs. A piezoelectric tube Figure 3 and a hollow ball are the main structural parts of the actuator.

The ball is a passive link of aluminium, steel or other material with high Young modulus. Several layers of distinct materials are a possible structural option of passive ball too. Single piezoelectric tube has to be modified in order to provide three permanent spinning axes. Therefore an internal electrode 
of the tube is separated into three equal sections. Driving teeth are employed to define accurate contact zones with the ball. Control techniques for multi-axis piezoelectric actuators are already defined [11].

The aim of this work is to determine inverse dynamics of TTR with two different spherical actuators. This robot has a maximum number of DOFs for 3D manipulation. The determination of robot dynamics also includes the estimation of Coriolis and centrifugal forces. These forces are hard to evaluate in analytical way. According to that is selected to use a universal and effective method of Euler-Lagrange equations and an analytic-potential model too. The results of preferred methods are almost equal, but their operational speeds are different.

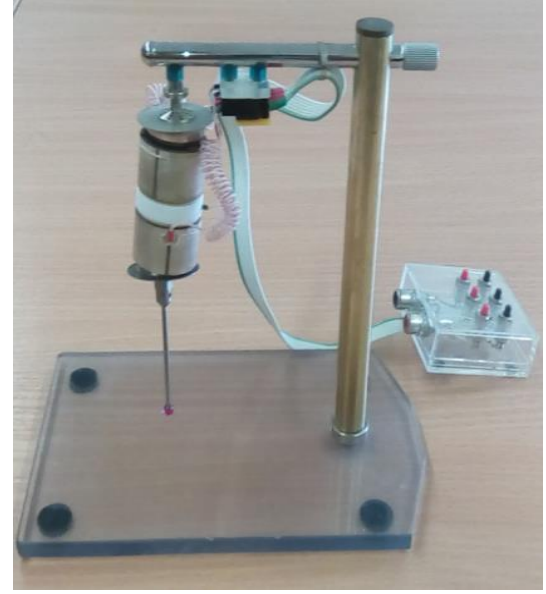

Figure 1. Piezorobot with 6 DOFs

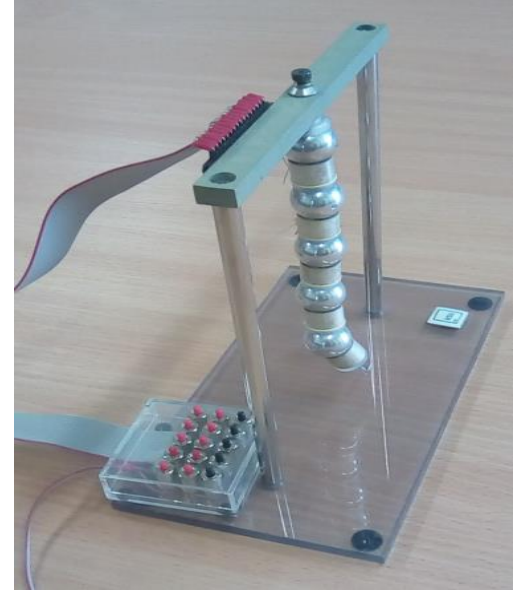

Figure 2. Piezorobot with 15 DOFs

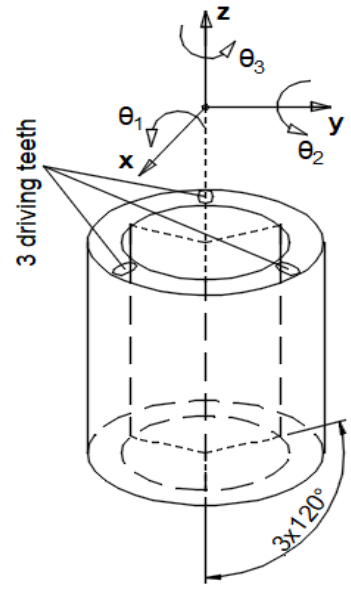

Figure 3. Structure of modified piezoelectric tube

\section{REVIEW OF EULER-LAGRANGE DYNAMICS}

Euler-Lagrange (E-L) method is one of the most universal and simplest ways to solve inverse dynamics of mechanical system. It lets use different coordinate systems (CSs) and consists of the highest integral members. Those are potential $P_{i}$ and kinetic $T_{i}$ energies of the links. E-L method is based on differential approach. Therefore it can provide all possible action and reaction forces or torques. It leads to a final form (1) [12].

$$
f=D(q) \cdot \ddot{q}+C(q, \dot{q}) \cdot \dot{q}+g(q)
$$

where: $f$ - vector of total torques for actuation of the DOFs; $q, \dot{q}$ - vectors of local robot coordinates and their first derivatives respectively; $D(q) \ddot{q}$-matrix of torques to evaluate inertial properties; $C(q, \dot{q}) \dot{q}-$ matrix of torques to estimate the effects of friction, damping and the influence of centrifugal and Coriolis forces also; $g(q)$ - matrix of torques to evaluate the effect of gravity and so on.

E-L method can be employed to determine the dynamics of systems with flexible links [13] and to implement various restrictions for its investigation too [14]. This method is selected to evaluate the torques of robot DOFs at picked time moments. The dependencies of the torques on time can be approximated too.

General Euler-Lagrange equation is presented in (2). Consistently a special variable La called Lagrangian is needed to estimate (3) [15]. A new and more comprehensive formula of E-L (4) can be written using the special features of Lagrangian. Kinetic energy of spinning link $i$ is evaluated by (5) and potential energy of the same link is determined by (6).

$$
\begin{aligned}
& f=\frac{d}{d t} \frac{\partial L a}{\partial \dot{q}}-\frac{\partial L a}{\partial q} \\
& L a=\sum_{i} T_{i}-\sum_{i} P_{i}
\end{aligned}
$$




$$
\begin{aligned}
& f=\frac{d}{d t} \frac{\partial T}{\partial \dot{q}}-\frac{\partial P}{\partial q} \\
& T_{i}=\operatorname{Trace}\left(\omega_{i} \omega_{i}^{T}\right) I_{i} / 2 \\
& P_{i}=-m_{i} g h_{i}
\end{aligned}
$$

where: $\frac{d}{d t}, \frac{\partial}{\partial q}-$ a single derivation on time and a partial derivation on local coordinate respectively; $L a-$ substitute of mechanical energy for investigated system (Lagrangian); $t$ - time; $T_{i}, P_{i}$ - kinetic and potential energies of link $i$ respectively; Trace()-sum of elements on the main diagonal of matrix; $\omega_{i}$-matrix of angular speed projections to the base CS regarding to link $i ; I_{i}, m_{i}, h_{i}$ - moment of inertia, mass and center height of the link in accordance to the grounded CS; $g$ - acceleration of gravity and so on.

The speed of any robot point can be estimated employing (7). The derivative of single orientation matrix can be determined using (8).

$$
\begin{aligned}
& \begin{array}{l}
v_{0}^{i}=\frac{d}{d t} r_{0}^{i}=\frac{d}{d t} O_{0}^{i} r_{i}=\frac{d}{d t}\left[A_{0}^{1} \cdot A_{1}^{2} \cdot \ldots \cdot A_{i-1}^{i} r_{i}\right] \\
\quad=\dot{A}_{0}^{1} A_{1}^{2} \cdot \ldots \cdot A_{i-1}^{i} r_{i}+A_{0}^{1} \dot{A}_{1}^{2} \cdot \ldots \cdot A_{i-1}^{i} r_{i}+\cdot \ldots \cdot A_{0}^{i} \dot{r}_{i}
\end{array} \\
& \begin{array}{l}
\frac{d}{d t} A_{i-1}^{i}=\frac{\partial A_{i-1}^{i}}{\partial \theta_{i}} \dot{\theta}_{i}
\end{array}
\end{aligned}
$$

where: $v_{0}^{i}$-global speed vector of selected link-point $i ; r_{0}^{i}, r_{i}$-global and initial position vectors of the link-point respectively; $O_{0}^{i}$-global orientation matrix of the link, which directly associates with point $i$; $A_{0}^{i}$-single orientation matrix from the first CS to the grounded CS; $\theta_{i}$-local coordinate (angle) of link $i$ and so on.

\section{PARAMETERS OF DYNAMICAL TASK}

A TTR with two different spherical actuators Figure 4 is selected to explore. A preferred spherical actuator consists of a modified piezoelectric tube with three evenly distributed internal electrodes in circumferential direction and a spherical segment. Three longitudinal zones of the tube can be excited independently, but generated displacements are partially dependent because of a monolith structure.

The kinematical lengths of the first $\left(\mathrm{OO}_{1}\right)$ and second $\left(\mathrm{O}_{1} \mathrm{O}_{2}\right)$ robot links are chosen to be equal to $40 \mathrm{~mm}$. The first link of the TTR is compounded of piezoelectric tube and two cut balls. External radiuses of the balls from steel are equal to $10 \mathrm{~mm}$ and their internal radiuses-9 $\mathrm{mm}$. An external diameter of the tube is $10 \mathrm{~mm}$, its internal diameter and length are 8 and $24 \mathrm{~mm}$ respectively. The second link of the TTR is made of piezoelectric tube too. Its length is $31 \mathrm{~mm}$ and it has the same cross-section dimensions. The tubes are made of PZT-4, which density is taken as $7.6 \mathrm{~g} / \mathrm{cm}^{3}$. The density of steel is selected as $7.8 \mathrm{~g} / \mathrm{cm}^{3}$. Therefore the masses of the first and second links are $21.7 \mathrm{~g}$ (9) and $6.7 \mathrm{~g}(10)$ respectively.

$$
\begin{aligned}
& m \cong 2\left(\frac{4}{3} \pi\left(R^{3}-r^{3}\right)-\frac{\pi D^{2}}{4}(R-r)\right) \rho_{s t}+\frac{\pi\left(D^{2}-d^{2}\right) l_{1}}{4} \rho_{P Z T} \\
& m_{2}=\frac{\pi\left(D^{2}-d^{2}\right) l_{2}}{4} \rho_{P Z T}
\end{aligned}
$$

where: $m_{1}, m_{2}$-masses of the first and second links respectively; $\pi$-Archimedes' constant; $R, r$-external and internal radiuses of the ball; $D, d$-external and internal diameters of piezoelectric tube; $\rho_{s t}, \rho_{P Z T}$-densities of steel and PZT-4 respectively; $l_{1}, l_{2}$, -lengths of the first and second piezoelectric tubes respectively.

A reference point of the grounded robot coordinate system (CS) is marked as O. A reference point of the first link CS is noted as $\mathrm{O}_{1}$ and the point of the second link CS - as $\mathrm{O}_{2}$. An axis $\mathrm{z}_{0}$ is parallel to the axis of gravity, an axis $z_{1}$-to a longitudinal symmetry axis of the first link and an axis $z_{2}$-to the symmetry axis of the second link. The first and second coordinate systems of the robot are rigidly attached to the first and second links respectively. A reference configuration of the robot can be notified as initial, when the same type axes are made parallel to each other $\left(\mathrm{x}_{0}-\mathrm{x}_{1}-\mathrm{x}_{2}, \mathrm{y}_{0}-\mathrm{y}_{1}-\mathrm{y}_{2}\right.$ and $\left.\mathrm{z}_{0}-\mathrm{z}_{1}-\mathrm{z}_{2}\right)$. The tube of the first spherical 
actuator is its primary link with direct control to its secondary link. The tube of the second spherical actuator is its secondary link with indirect motion. It provides two actions at once: excitation and movement. The orientations of tube axes are permanent, because its geometry is static. Therefore the first spherical actuator can be referenced as direct type and the second spherical actuator-as inverse type [16] with rotational axes $\mathrm{x}_{2}-\mathrm{y}_{2}-\mathrm{Z}_{2}$ placed on $\mathrm{O}_{1}$ reference point. This repetition of $\mathrm{O}_{2}$ coordinate system is important to ensure the rightness of actuating. Consistently evaluation methods of orientations for movable links are going to be different.

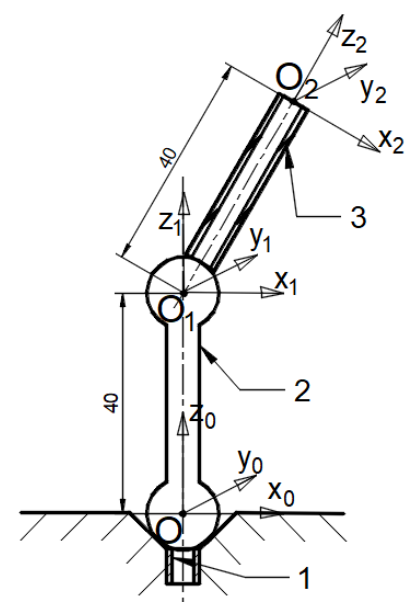

Figure 4. TTR with two different spherical actuators

Seeking to properly determine an orientation matrix of the selected link is important to evaluate its actuation sequence. To realize a hybrid rotational axis and to ensure a proportional excitation of selected DOFs is a very complicated technical task, because of finite stiffness of the ball, friction dependency on speed, losing contact pair or the action of external torque and so on. The manipulation of real spherical actuator can be executed more accurately only if its DOFs are going to be activated separately. This statement can be proved regarding to a simpler control technique, which requires a lower knowledge of experimental testing. To implement casual orientations for robot links are picked to spin the first link about $\mathrm{x}_{0}$ and $\mathrm{y}_{0}$ axes by angles 0.12 and 0.15 rad respectively and to spin the second link about $\mathrm{x}_{2}$ and $\mathrm{y}_{2}$ axes by angles -0.1 and -0.15 rad respectively. To realize a next robot configuration is selected to activate only $\mathrm{x}$ type of axes. Every angular speed dependence of $\mathrm{x}$ type axes on time is chosen to be a triangle type Figure 5. Amplitudes of global angular speeds for axes $\mathrm{x}_{0}$ and $\mathrm{x}_{2}$ are selected to be 0.1 and $-0.05 \mathrm{rad} / \mathrm{s}$ respectively. Both DOFs are going to be started at the same time and their durations are $2 \mathrm{~s}$. The maximum sizes of local angular speeds for movable links are equal to their magnitudes of permanent accelerations ( 0.1 and $-0.15 \mathrm{rad} / \mathrm{s}^{2}$ respectively). The last parameters can be easily estimated and are important for estimation of kinetic torques in robot dynamics employing an analytical way.

A center of link mass is taken as a middle point of its longitudinal symmetry line, because each robot link is a rod type. A mass moment of inertia in accordance to parallel axes of body is presented in (11). The first link moment of inertia about $\mathrm{x}_{1}$ or $\mathrm{y}_{1}$ axes can be evaluated by (12) and is $16.6 \cdot 10^{-6} \mathrm{~kg} \cdot \mathrm{m}^{2}$. Its moment of inertia about $\mathrm{z}_{1}$ axis is determined by (13) and is equal to $1.4 \cdot 10^{-6} \mathrm{~kg} \cdot \mathrm{m}^{2}$. The second link moment of inertia about $x_{2}$ or $y_{2}$ axes can be estimated with (14) and is $4.6 \cdot 10^{-6} \mathrm{~kg} \cdot \mathrm{m}^{2}$. Its moment of inertia about $\mathrm{z}_{2}$ axis is determined by (15) and is equal to $0.6 \cdot 10^{-6} \mathrm{~kg} \cdot \mathrm{m}^{2}$.

$$
\begin{aligned}
& I_{p_{\_} a}=I_{c_{\_} a}+m d^{2} \\
& I_{1 \_x}=I_{1 \_y}=m_{1 \_t} l_{1}^{2} / 12+m_{1 \_t} d_{1}^{2}+2 m_{s p} \frac{2\left(R^{5}-r^{5}\right)}{5\left(R^{3}-r^{3}\right)}+m_{s p} L_{1}^{2} \\
& I_{1 \_z}=m_{1 \_t}\left(D^{2}+d^{2}\right) / 2+2 m_{s p} \frac{2\left(R^{5}-r^{5}\right)}{5\left(R^{3}-r^{3}\right)} \\
& I_{2 \_x}=I_{2 \_y}=m_{2} l_{2}^{2} / 12+m_{2} d_{2}^{2}
\end{aligned}
$$




$$
I_{2 \_z}=m_{2}\left(D^{2}+d^{2}\right) / 2
$$

where: $I_{c_{-} a}, I_{p_{-} a}-$ mass moments of inertia in accordance to center axis and parallel moved axis of body respectively; $m$ - body mass; $d$ - normal distance between the axes of inertia; $I_{1 \_x}, I_{2 \_} x$ - mass moments of inertia about $\mathrm{x}$ axes of the first and second links respectively; $m_{1-t}-$ mass of the first link tube $(5.2 \mathrm{~g})$; $d_{1}$-distance between inertial axes of the first link $(20 \mathrm{~mm}) ; m_{s p}$-mass of the hollow ball $(8.3 \mathrm{~g})$; $L_{1}$-kinematical length of the first link $(40 \mathrm{~mm}) ; d_{2}$-distance between inertial axes of the second link $(24.5$ $\mathrm{mm})$ and so on.

The first link of the TTR is able to turn only about the axes of coordinate system O. This means that any spinning axis of the CS is going to be a hybrid one in accordance to principal axes of the first link. An analytic-geometric approach can be selected to evaluate a mass moment of inertia for free chosen axis (MIFCA).

A freely selected axis OL of inertia has to cross a mass center of body $\boldsymbol{K}$ and be expressed by three spatial angles $\alpha, \beta, \gamma$ in accordance to its principal axes $x, y$ and $z$ Figure 6. A general formula of the MIFCA is introduced in (16). Centrifugal moments $I_{y z}, I_{x z}$ and $I_{x y}$ of the body are equal to 0 , because it is fully symmetric and isotropic. Now it is important to mention an orientation matrix (17) of body $\boldsymbol{K}$ according to the base CS. Then mass moments of inertia for the body can be apparently determined regarding to the main CS axes (18-20). If the moment of robot link is estimated in accordance to earlier spherical joint, then it is important to evaluate the additional inertial quantity of shifted axes.

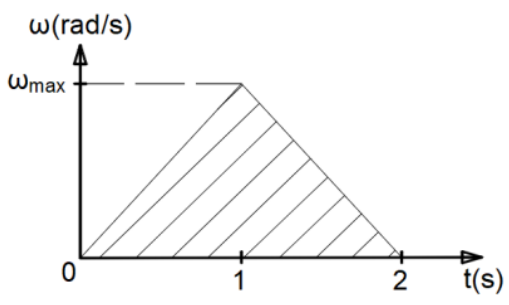

Figure 5. Shape of angular speed dependence on time

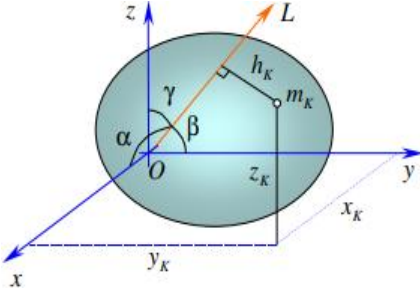

Figure 6. Mass moment of inertia for free selected axis

$$
\begin{aligned}
& I_{O L}=\begin{array}{c}
(\cos \alpha)^{2} I_{x}+(\cos \beta)^{2} I_{y}+(\cos \gamma)^{2} I_{z}-2 \cos \beta \cos \gamma I_{y z}-2 \cos \gamma \cos \alpha I_{x z} \\
-2 \cos \alpha \cos \beta I_{x y}
\end{array} \\
& O_{0}^{K}=\left[\begin{array}{lll}
x_{x_{0}} & y_{x_{0}} & z_{x_{0}} \\
x_{y_{0}} & y_{y_{0}} & z_{y_{0}} \\
x_{z_{0}} & y_{z_{0}} & z_{z_{0}}
\end{array}\right] \\
& I_{K_{-} x_{0}}=\left(x_{x_{0}}\right)^{2} I_{x}+\left(y_{x_{0}}\right)^{2} I_{y}+\left(z_{x_{0}}\right)^{2} I_{z} \\
& I_{K_{-} y_{0}}=\left(x_{y_{0}}\right)^{2} I_{x}+\left(y_{y_{0}}\right)^{2} I_{y}+\left(z_{y_{0}}\right)^{2} I_{z} \\
& I_{K_{-} z_{0}}=\left(x_{z_{0}}\right)^{2} I_{x}+\left(y_{z_{0}}\right)^{2} I_{y}+\left(z_{z_{0}}\right)^{2} I_{z}
\end{aligned}
$$

where: $I_{O L}$-mass moment of inertia for body $\boldsymbol{K}$ regarding to a selected axis OL; $\alpha-$ angle between a spinning axis OL and a principal axis $\mathrm{x}$ of the body; $I_{x}, I_{y}, I_{z}$ - principal moments of inertia for body $\boldsymbol{K} ; x_{x_{0}}$, $y_{x_{0}}, z_{x_{0}}$-projections of principal axes $\left(x, y\right.$ and $z$ ) to a base axis $x_{0} ; I_{K_{-} x_{0}}, I_{K_{-} y_{0}}, I_{K_{-} z_{0}}-$ mass moments of inertia for the body according to the main CS axes $\left(\mathrm{x}_{0}-\mathrm{y}_{0}-\mathrm{z}_{0}\right)$ and so on.

\section{APPLICATION OF EULER-LAGRANGE DYNAMICS}

An initial orientation of the first link is estimated by (21) using Roll-Pitch-Yaw method and the orientation of the second link-by (22) [17]. The last equation can be proved employing orthogonal and transposition features of matrices. An orientation of the first link at the first and second halves of actuation can be evaluated by (23 and 25) respectively. An orientation of the second link at the first and second halves of actuation can be determined by (24 and 26) respectively. In order to estimate a TTR control function of any DOF is selected to use three main time points (initial, medium and final) of its excitation period. 
Local speed dependencies on time of the first link can be determined employing these forms: $\omega_{1 x}(0 \leq \Delta t \leq 1)=\varepsilon_{1 F H} \Delta t, \omega_{1 x}(1 \leq \Delta t \leq 2)=\Omega_{1 x}-\varepsilon_{1 S H} \Delta t, \omega_{1 x}(\Delta t \geq 2)=0$. The speed dependencies of the second link can be written using the same way. Global speeds of the first and second halves regarding to the first link are estimated by (27-28). The speeds of the first and second halves according to the second link are evaluated by (29-30).

$$
\begin{aligned}
& O_{0}^{1}=A_{y} \cdot A_{x} \\
& O_{1}^{2}=\left(A_{y} \cdot A_{x}\right)^{-1}=A_{x}^{T} \cdot A_{y}^{T} \\
& O_{0}^{1}(t<1 s)=A_{x}\left(\varepsilon_{1 x} \cdot t^{2} / 2\right) \cdot A_{y}\left(\theta_{1 y}\right) \cdot A_{x}\left(\theta_{1 x}\right) \\
& O_{0}^{2}(t<1 s)=A_{x}\left(\varepsilon_{1 x} \cdot t^{2} / 2\right) \cdot A_{y}\left(\theta_{1 y}\right) \cdot A_{x}\left(\theta_{1 x}\right) \cdot A_{x}^{T}\left(\theta_{2 x}\right) \cdot A_{y}^{T}\left(\theta_{2 y}\right) \cdot A_{x}^{T}\left(\varepsilon_{2 x} \cdot t^{2} / 2\right) \\
& O_{0}^{1}(t>1 s)=A_{x}\left(0.05+0.1-\varepsilon_{1 x} \cdot(t-1)^{2} / 2\right) \cdot A_{y}\left(\theta_{1 y}\right) \cdot A_{x}\left(\theta_{1 x}\right) \\
& O_{0}^{2}(t>1 s)=A_{x}\left(0.05+0.1 \cdot t-\varepsilon_{1 x} \cdot(t-1)^{2} / 2\right) \cdot A_{y}\left(\theta_{1 y}\right) \cdot A_{x}\left(\theta_{1 x}\right) \cdot A_{x}^{T}\left(\theta_{2 x}\right) \\
& \omega_{1 F H}=\frac{\partial O_{0}^{1}}{\partial \varepsilon_{1 x}} / t^{2} \cdot 2 \cdot\left(\varepsilon_{1 x} t\right) \cdot A_{x}^{T}\left(-0.075-0.15 \cdot t-\varepsilon_{2 x} \cdot(t-1)^{2} / 2\right) \\
& \omega_{1 S H}=\frac{\partial O_{0}^{1}}{\partial \varepsilon_{1 x}} /-(t-1)^{2} \cdot 2 \cdot\left(\Omega_{1 x}-\varepsilon_{1 x}(t-1)\right) \\
& \omega_{2 F H}=\frac{\partial O_{0}^{2}}{\partial \varepsilon_{1 x}} / t^{2} \cdot 2 \cdot\left(\varepsilon_{1 x} t\right)+\frac{\partial O_{0}^{2}}{\partial \varepsilon_{2 x}} / t^{2} \cdot 2 \cdot\left(\varepsilon_{2 x} t\right) \\
& \omega_{2 S H}=\frac{\partial O_{0}^{2}}{\partial \varepsilon_{1 x}} /-(t-1)^{2} \cdot 2 \cdot\left(\Omega_{1 x}-\varepsilon_{1 x}(t-1)\right) \\
& \quad+\frac{\partial O_{0}^{2}}{\partial \varepsilon_{1 x}} /-(t-1)^{2} \cdot 2 \cdot\left(\Omega_{2 x}-\varepsilon_{2 x}(t-1)\right)
\end{aligned}
$$

where: $A_{x}$-single orientation matrix about axis x; $A_{x}^{T}$-transpose matrix of $A_{x} ; O_{1}^{2}$-orientation matrix from the second CS to the first CS; $\varepsilon_{1 x}, \varepsilon_{2 x}$-variables of angular accelerations about $\mathrm{x}$ axes for the first and second links respectively; $\theta_{1 x}, \theta_{1 y}$-selected angular movements of the first link about $\mathrm{x}_{0}$ and $\mathrm{y}_{0}$ axes $(0.12$ and $0.15 \mathrm{rad}$ respectively); $\omega_{1 F H}, \omega_{1 S H}$-angular speeds of the first link at the first and second halves respectively; $\Omega_{1 x}, \Omega_{2 x}$-amplitudes of global angular speeds about axes $\mathrm{x}_{0}$, (replaced) $\mathrm{x}_{2}$ respectively and so on.

A total kinetic energy of the system is estimated employing (31). Kinetic energy of the second link is determined by (31) and kinetic energy of the first link-by (33). A mass moment of inertia for the first link about axis $\mathrm{x}_{0}$ is evaluated by (34) and the moment of the second link about $\mathrm{x}_{0}$-by (35).

$$
\begin{aligned}
& T=T_{1}+T_{2} \\
& T_{2}=\operatorname{Trace}\left(\omega_{2 x} \cdot \omega_{2 x}^{T}\right) \cdot\left(I_{2 \_x_{2}} / 2\right) / 2 \\
& T_{1}=\operatorname{Trace}\left(\omega_{1 x} \cdot \omega_{1 x}^{T}\right) \cdot\left(I_{1 \_x_{0}} / 2+I_{2 \_x_{0}} / 2\right) / 2 \\
& I_{1 \_x_{0}}=\left(O_{0}^{1}(1.1)\right)^{2} \cdot I_{1 \_x_{1}}+\left(O_{0}^{1}(1.2)\right)^{2} \cdot I_{1 \_y_{1}}+\left(O_{0}^{1}(1.3)\right)^{2} \cdot I_{1 \_} z_{1} \\
& I_{2 \_x_{0}}=\left(O_{0}^{2}(1,1)\right)^{2} \cdot I_{2_{x_{2}}}+\left(O_{0}^{2}(1,2)\right)^{2} \cdot I_{2 y_{2}}+\left(O_{0}^{2}(1,3)\right)^{2} \cdot I_{2_{z_{2}}} \\
& \quad+m_{2}\left(\left(O_{0}^{1}(3.3) \cdot L_{1}\right)^{2}+\left(O_{0}^{1}(2.3) \cdot L_{1}\right)^{2}\right)
\end{aligned}
$$

where: $T_{1}, T_{2}$ - kinetic energies of the first and second links respectively; $I_{I_{-} x 0}, I_{2_{-} x 0}$-mass moments of inertia of the first and second links respectively in accordance to $\mathrm{x}_{0}$ axis and so on.

The total potential energy of the robot is estimated with (36). The potential energies of the first and second links are determined by (37-38) respectively.

$$
P=P_{1}+P_{2}
$$




$$
\begin{aligned}
& P_{1}=-m_{1} g\left(O_{0}^{1}(3.3) \cdot L_{1} / 2\right) \\
& P_{2}=-m_{2} g\left(O_{0}^{1}(3.3) \cdot L_{1}+O_{0}^{2}(3.3) \cdot L_{2} / 2\right)
\end{aligned}
$$

where: $P_{1}, P_{2}$-potential energies of the first and second links respectively; $L_{2}$-kinematical length of the second link $(40 \mathrm{~mm})$ and so on.

Dynamical results are estimated and presented in Table 1 and Figure 7 in regard to Euler-Lagrange method and selected parameters of motion.

Table 1. Dynamical results of the TTR

\begin{tabular}{clcc}
\hline Time moment $(\mathrm{s})$ & Quantity & $M 1(\mathrm{Nm})$ & $M 2(\mathrm{Nm})$ \\
\hline \multirow{2}{*}{$\mathbf{0}$} & Total & $\mathbf{1 1 , 1 2 \cdot 1 0 ^ { - 4 }}$ & $\mathbf{- 2 , 8 4 4 \cdot 1 0 ^ { - 4 }}$ \\
& Potential & $11,08 \cdot 10^{-4}$ & $-2,837 \cdot 10^{-4}$ \\
& Kinetic & $3,781 \cdot 10^{-6}$ & $-6,697 \cdot 10^{-7}$ \\
$\mathbf{0 , 3 3}$ & Potential & $11,62 \cdot 10^{-4}$ & $-3,006 \cdot 10^{-4}$ \\
$\mathbf{0 , 6 7}$ & Potential & $13,30 \cdot 10^{-4}$ & $-3,532 \cdot 10^{-4}$ \\
& Total & $\mathbf{1 5 , 8 9 \cdot 1 0 ^ { - 4 }}$ & $\mathbf{- 4 , 3 5 0 \cdot 1 0 ^ { - 4 }}$ \\
$\mathbf{1 , 0 0}$ & Potential & $16,00 \cdot 10^{-4}$ & $-4,370 \cdot 10^{-4}$ \\
& Kinetic & $-11,34 \cdot 10^{-6}$ & $2,009 \cdot 10^{-6}$ \\
$\mathbf{1 , 3 3}$ & Potential & $18,67 \cdot 10^{-4}$ & $-5,188 \cdot 10^{-4}$ \\
$\mathbf{1 , 6 7}$ & Potential & $20,30 \cdot 10^{-4}$ & $-5,680 \cdot 10^{-4}$ \\
& Total & $\mathbf{2 0 , 8 2} \cdot \mathbf{1 0}-\mathbf{4}$ & $\mathbf{- 5 , 8 3 6} \cdot \mathbf{1 0}-\mathbf{4}$ \\
$\mathbf{2 , 0 0}$ & Potential & $20,82 \cdot 10^{-4}$ & $-5,836 \cdot 10^{-4}$ \\
& Kinetic & 0 & 0 \\
\hline
\end{tabular}

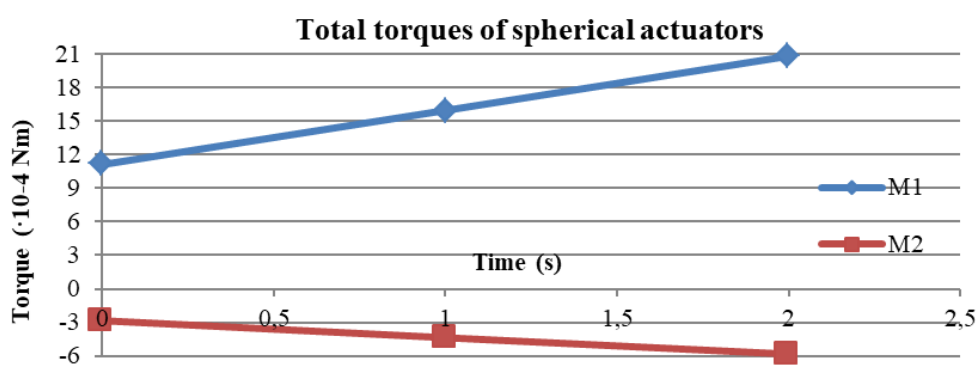

where: M1, M2-total torques about $\mathrm{x}$ axes of the first and second spherical actuators respectively

Figure 7. Dependencies of total torque on time

\section{DYNAMICS OF ANALYTICAL-POTENTIAL METHOD}

Analytical-potential method has been selected to assure the correctness of described E-L algorithm and its results. The orientations of robot links at the initial, medium and final configurations are determined by (23-26) too. The control of the first link is based on the main coordinate system $\mathrm{O}$. Its $\mathrm{z}_{0}$ axis is coincident with a gravitational axis. Therefore an evaluation of potential torque for the first SPA is quite simple (39). A value of torque about $\mathrm{x}$ axis is rated as positive, when the direction of links y projection goes from $\mathrm{y}$ axis to $\mathrm{z}$ axis (upward in the main quarter of $\mathrm{y}-\mathrm{z}$ plane or downward in $-\mathrm{y}-\mathrm{z}$ plane). Every single magnitude of potential torque should be multiplied by -1 in order to realize an opposite direction of gravitational force or the sense (negative to positive) of its elbow.

$$
M_{1 \_x}=-\left(m_{a} g O_{0}^{1}(2.3) \cdot L_{1} / 2+m_{2} g\left(O_{0}^{1}(2.3) \cdot L_{1}+O_{0}^{2}(2.3) \cdot L_{2} / 2\right)\right)
$$

where: $M_{1-x}$-torque about $\mathrm{x}_{0}$ axis because of gravity.

To evaluate a potential torque of the second link firstly the orientation of spinning axis $\mathrm{x}_{2}$ to the global area $\mathrm{x}_{0}-\mathrm{y}_{0}$ has to be determined. This can be done employing arctangent function (40-42), which depends on projections senses of mentioned axes Figure 8. Then an apparent torque (43) of the link and its orientation (44-46) to global area $\mathrm{x}_{0}-\mathrm{y}_{0}$ have to be estimated. Finally a perpendicularity of apparent torque to axis $\mathrm{x}_{2}$ can be evaluated and a perpendicularity of the same axis to a gravitational axis $\mathrm{z}_{0}$ too. The last two steps are essential in the estimation of the torque about a replaced axis $\mathrm{x}_{2}$ (47). 


$$
\begin{aligned}
& S_{1,1}=\operatorname{arctg}\left(O_{0}^{2}(1.1) / O_{0}^{2}(2.1)\right), \text { if } O_{0}^{2}(1.1)>0 \text { and } O_{0}^{2}(2.1)>0 \\
& S_{1,2}=\pi+\operatorname{arctg}\left(O_{0}^{2}(1.1) / O_{0}^{2}(2.1)\right), \\
& \text { if } O_{0}^{2}(1.1)>0 \text { and } O_{0}^{2}(2.1)<0 \text { or } O_{0}^{2}(1.1), O_{0}^{2}(2.1)<0 \\
& S_{1,3}=2 \pi+\operatorname{arctg}\left(O_{0}^{2}(1.1) / O_{0}^{2}(2.1)\right), \text { if } O_{0}^{2}(1.1)<0 \text { and } O_{0}^{2}(2.1)>0 \\
& N_{2 \_x}=m_{2} g\left(\left(\left(O_{0}^{2}(1.3)\right)^{2}+\left(O_{0}^{2}(2.3)\right)^{2}\right)^{1 / 2} \cdot L_{2} / 2\right) \\
& S_{2,1}=\operatorname{arctg}\left(O_{0}^{2}(1.3) / O_{0}^{2}(2.3)\right), \text { if } O_{0}^{2}(1.3)>0 \text { and } O_{0}^{2}(2.3)>0 \\
& S_{2,2}=\pi+\operatorname{arctg}\left(O_{0}^{2}(1.3) / O_{0}^{2}(2.3)\right), \\
& \text { if } O_{0}^{2}(1.3)>0 \operatorname{and} O_{0}^{2}(2.3)<0 \text { or } O_{0}^{2}(1.3), O_{0}^{2}(2.3)<0 \\
& S_{2,3}=2 \pi+\operatorname{arctg}\left(O_{0}^{2}(1.3) / O_{0}^{2}(2.3)\right), \text { if } O_{0}^{2}(1.3)<0 \text { and } O_{0}^{2}(2.3)>0 \\
& M_{2 \_x}=-N_{2 \_} \cdot \sin \left(S_{1, j}-S_{2, j}\right) \cdot\left(\left(O_{0}^{2}(1.1)\right)^{2}+\left(O_{0}^{2}(2.1)\right)^{2}\right)^{1 / 2}
\end{aligned}
$$

where: $S_{i, j}$-arctangent function to estimate the direction ( $i$-mark for sense of orientation and $j$-index for applicable function); $N_{2 \_}, M_{2 \_}-$apparent and real torques about a replaced axis $\mathrm{x}_{2}$ because of gravity and so on.

Numerical and graphical results are generated Table 2, Figure 9 in accordance to a recently described method of inverse dynamics. It is based on the action of gravity regarding to the adequate parameters of motion.

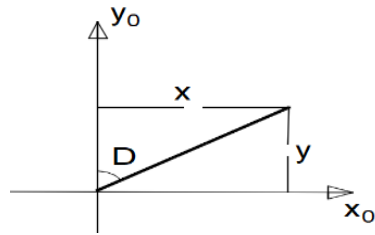

Figure 8. Projection of robot link or axis in area $\mathrm{x}_{0}-\mathrm{y}_{0}$
Table 2. Results of the analytic-potential research

\begin{tabular}{ccc}
\hline Time moment $(\mathrm{s})$ & $M 1 *(\mathrm{Nm})$ & $M 2 *(\mathrm{Nm})$ \\
\hline $\mathbf{0}$ & $11,08 \cdot 10^{-4}$ & $-2,837 \cdot 10^{-4}$ \\
$\mathbf{0 , 3 3}$ & $11,62 \cdot 10^{-4}$ & $-3,006 \cdot 10^{-4}$ \\
$\mathbf{0 , 6 7}$ & $13,30 \cdot 10^{-4}$ & $-3,532 \cdot 10^{-4}$ \\
$\mathbf{1 , 0 0}$ & $16,00 \cdot 10^{-4}$ & $-4,370 \cdot 10^{-4}$ \\
$\mathbf{1 , 3 3}$ & $18,67 \cdot 10^{-4}$ & $-5,188 \cdot 10^{-4}$ \\
$\mathbf{1 , 6 7}$ & $20,30 \cdot 10^{-4}$ & $-5,680 \cdot 10^{-4}$ \\
$\mathbf{2 , 0 0}$ & $20,82 \cdot 10^{-4}$ & $-5,836 \cdot 10^{-4}$ \\
\hline
\end{tabular}

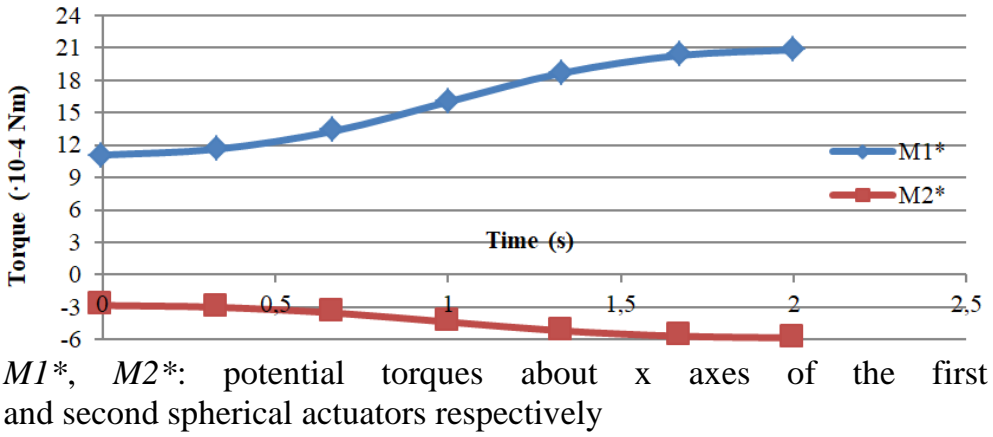

Figure 9. Dependencies of potential torque on time

\section{CONCLUSION}

The results of Euler-Lagrange dynamics are partly confirmed by the results of analytic-potential method. Kinetic torques of E-L method can be confirmed by theoretical way too. Actuators dependencies of total torque on large time steps can be approximated as linear graphs. Actuators dependencies of potential torque on smaller time steps are similar shapes to links dependencies of position. Kinetic torques are more than 100 times smaller than potential ones regarding to adequate input parameters. The calculation with MATLAB software of 14 potential torques according to E-L method lasts about $2.8 \mathrm{~s}$ and an analogous evaluation regarding to analytic-potential method takes about $3.1 \mathrm{~s}$. The estimation with the same software of 6 potential and 6 kinetic torques in regard to Euler-Lagrange method lasts about $5.3 \mathrm{~s}$. A casual E-L method can be simplified or changed into the analytical-potential method seeking to improve coding speed and to accelerate numerical operations of dynamical analysis for a gently trunk type robot. 


\section{ACKNOWLEDGEMENTS}

The work was supported by the Research Council of Lithuania under the project SmartTrunk, No. MIP-084/2015

\section{REFERENCES}

[1] J. Li, et al., "Autonomous Continuum Grasping," International Conference on Intelligent Robots and Systems, pp. 4569-4576, 2014.

[2] T. Mahl, et al., "Forward Kinematics of a Compliant Pneumatically Actuated Redundant Manipulator," IEEE Conference on Industrial Electronics and Applications, pp. 1267-1273, 2012.

[3] A. Nagarajan, S. K. R. Kanna and V. M. Kumar, "Multibody dynamic simulation of a hyper redundant robotic manipulator using ADAMS ansys interaction," International Conference on Algorithms, Methodology, Models and Applications in Emerging Technologies (ICAMMAET), pp. 1-6, Chennai, 2017.

[4] S. E. Tabatabaie and M. Shahinpoor, "Artificial Soft Robotic Elephant Trunk Made with Ionic Polymer-Metal Nanocomposites (IPMCs)," International Robotics \& Automation Journal, vol. 5, no.4, pp. 138-142, 2019.

[5] B. A. Jones and I. D. Walker, "Kinematics for multisection continuum robots," in IEEE Transactions on Robotics, vol. 22, no. 1, pp. 43-55, Feb. 2006.

[6] W. Chung, C. Rhee, Y. Shim, H. Lee and S. Park, "Door-Opening Control of a Service Robot Using the Multifingered Robot Hand," in IEEE Transactions on Industrial Electronics, vol. 56, no. 10, pp. 3975-3984, Oct. 2009.

[7] O. Salomon and A. Wolf, "Inclined Links Hyper-Redundant Elephant Trunk-Like Robot," Mechanisms and Robotics, vol. 4, no. 4, 2012.

[8] P. Liljeback, I. U. Haugstuen and K. Y. Pettersen, "Path Following Control of Planar Snake Robots Using a Cascaded Approach," in IEEE Transactions on Control Systems Technology, vol. 20, no. 1, pp. 111-126, Jan. 2012.

[9] E. Kelasidi, et al., "Locomotion Efficiency Optimization of Biologically Inspired Snake Robots," Applied Sciences, vol. 8, no. 1, 2018.

[10] R. C. Luo and C. Kuo, "Intelligent Seven-DoF Robot With Dynamic Obstacle Avoidance and 3-D Object Recognition for Industrial Cyber-Physical Systems in Manufacturing Automation," in Proceedings of the IEEE, vol. 104, no. 5, pp. 1102-1113, May 2016.

[11] R. Bansevičius and V. Kargaudas, "Attitude Control of Micro-and Nanosatellites Using Multi-Degree-of-Freedom Piezoelectric Actuators," Vibration problems ICOVP, vol. 139, pp. 379-384, 2011

[12] J. Iqbal, "Modern Control Laws for an Articulated Robotic Arm: Modeling and Simulation," Engineering, Technology \& Applied Science Research, vol. 9, no. 2, pp. 4057-4061, 2019.

[13] Chunxia Z., et al., "Effect of Links Deformation on Motion Precision of Parallel Manipulator Based on Flexible Dynamics," Industrial Robot, vol. 44, no. 6, pp. 776-787, 2017.

[14] D. Dopico, et al., "Direct Sensitivity Analysis of Multibody Systems with Holonomic and Nonholonomic Constraints via an Index-3 Augmented Lagrangian Formulation with Projections," Nonlinear Dynamics, vol. 93, pp. 2039-2056, 2018.

[15] D. Baleanu, et al., "The Motion of a Bead Sliding on a Wire in Fractional Sense," Acta Physica Polonica A, vol. 131, vol. 6, pp. 1561-1564, 2017.

[16] A. Augustaitis, et al., "Kinematics of Trunk-Like Robots with Piezo Actuators," Mechanics, vol. 24, no. 2, pp. 254-259, 2018.

[17] Y. H. Hwang, et al., "An Electrorheological Spherical Joint Actuator for a Haptic Master with Application to Robot-Assisted Cutting Surgery," Sensors and Actuators A: Physical, vol. 249, pp. 163-171, 2016.

\section{BIOGRAPHIES OF AUTHORS}

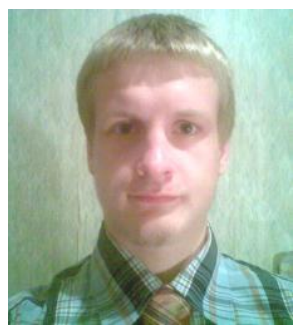

Aistis Augustaitis received BS degree in Transport Engineering from Kaunas University of Technology (KTU) in 2014, MS degree in Mechanical Engineering from KTU in 2016. Currently he is studying for $\mathrm{PhD}$ in Mechanical Engineering at Vilnius Gediminas Technical University (VGTU). His current research interests include the trajectory planning and control of trunk-type robots with emphasis on numerical analysis.

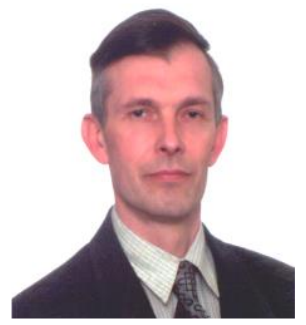

Vytautas Jurènas graduated from Kaunas University of Technology (KTU) in 1979 and received degree in Mechanical Engineering. He received PhD from KTU in 1993 in the field of Mechanical Engineering. He is a senior researcher in KTU Mechatronics Institute. His research interests include piezomechanics and diagnostics of mechanical systems.

Int J Rob \& Autom, Vol. 9, No. 2, June 2020 : 113 - 122 